\title{
RESONANT-HOLOGRAPHIC-INTERFEROMETRY FOR ABSOLUTE MEASUREMENTS OF EXCIMER LASER-ABLATED NEUTRAL-ATOM PLUME LINE-DENSITY PROFILES
}

\author{
R. M. Gilgenbach, R. A. Lindley, C. H. Ching, and J. S. Lash \\ Intense Energy Beam Interaction Laboratory \\ Nuclear Engineering Department \\ University of Michigan, Ann Arbor, MI 48109-2104
}

\begin{abstract}
Experiments have been performed to measure Al neutral atom absolute line-density profiles using resonant-holographic-interferometry. The ablation source is a $\mathrm{KrF}$ excimer laser with a per-pulse energy of about $0.8 \mathrm{~J}$. Targets are either pure aluminum or $\mathrm{Al}_{2} \mathrm{O}_{3}$. Aluminum groundstate neutral atom line-densities are probed by a dye laser tuned near the $394.401 \mathrm{~nm}$ line. A double-pulse interferometry technique is employed in which one pulse includes the laser ablation plume and a second pulse generates reference fringes on a holographic plate by rotating a mirror. Holograms are reconstructed to give interference fringes on film by using a helium-neon laser. Interferograms of laser ablated Al metal give maximum Al neutral plume line-densities in the range of 4-10 $\times 10^{14} \mathrm{~cm}^{-2}$. Aluminum neutral line-densities from $\mathrm{Al}_{2} \mathrm{O}_{3}$ targets are as much as $10-20$ times larger than from $\mathrm{Al}$ metal targets. The sensitivity of this resonant diagnostic is 4 to 5 orders of magnitude higher than nonresonant neutral-particle interferometry and may be adjusted by tuning the dye laser wavelength shift away from the resonance line. This diagnostic has been demonstrated in vacuum, gas (14 mTorr - 35 Torr) and RF plasma (30 mTorr - 1 Torr) environments. At higher gas pressures, the nonresonant gas-dynamic fringe shift can be subtracted to give the resonant fringe shift and species density.
\end{abstract}

\section{INTRODUCTION}

A crucial problem in laser-ablative machining, thin-film deposition, and $X$-ray production is the measurement of particlespecies density profiles. It is advantageous to make these density profile measurements in a single plume pulse. Researchers at other laboratories ${ }^{1-7}$ have employed laser-induced-fluorescence ${ }^{1,5}$, direct particle detection ${ }^{2}$, optical absorption ${ }^{3}$ and emission spectroscopy 4 of atoms and ions. Hook spectroscopy has been employed by others ${ }^{5}$ to measure plume species line-density along a laser beam path. Fast-photography has also been used to follow plume dynamics. 6,7 
Experimental research at the University of Michigan has been directed toward laser ablation diagnostics which characterize the time-resolved behavior of the plume in a single pulse.8-14 Dye-laser-resonance-absorption-photography, (DLRAP), is a speciesresolved diagnostic, first applied to laser-ablation at UM. 8,9 This diagnostic utilizes the absorption due to the imaginary part of the index of refraction at an atomic or molecular resonance line. DLRAP has been demonstrated for measurements of plume atomic 8 and molecular ${ }^{8}$ species expansion velocities and hydrodynamics in vacuum and gas environments.

The most powerful species-resolving laser ablation diagnostic investigated at $U M$ is resonant-holographic-interferometry $(\mathrm{RHI})^{14}$. The RHI diagnostic measures the phase shift of laser light passing through an ablation plume at a laser wavelength close $( \pm 0.006-0.02 \mathrm{~nm})$ to an atomic resonance line. The image of the plume phase-shift is recorded by a double-pulsed dye laser on a holographic plate. The reconstructed hologram yields an interferogram on which the fringe shift is directly proportional to the plume single-species line-density, in this research, aluminum ground-state neutrals.

\section{EXPERIMENTAL CONFIGURATION}

The configuration of the experiment is depicted in simplified form in Figure 1. A KrF laser with an output energy of about $0.8 \mathrm{~J}$ is focused at normal incidence on the ablation target, which is either $98.5 \%$ pure aluminum or $\mathrm{Al}_{2} \mathrm{O}_{3}(>99.5 \%$ pure). The diagnostic dye laser is a Lambda Physik FL3002, pumped by a $\mathrm{XeCl}$ laser. In these experiments, the dye laser is tuned near the $394.401 \mathrm{~nm}$ aluminum ground-state neutral transition. A quartz inteferometry flat splits the beam into object and reference paths which are expanded to a large diameter $(7.5 \mathrm{~cm})$ in order to illuminate the entire plume. The path lengths of the two beams are matched to within the estimated coherence length $(\sim 5 \mathrm{~cm})$ of the dye laser. The double-pulse holographic interferometry technique is employed, in which one pulse illuminates the ablation plume and the second pulse (without the plume) includes a slight mirror rotation, which introduces the interference fringes. This technique allows inexpensive optics to be used. The two laser beams are recombined at $8^{\circ}$ angular difference on a holographic plate chosen for its sensitivity at $394.4 \mathrm{~nm}$. Four 1.5"X 1.5", double-pulse holograms can be taken on a single $4 \times 5$ inch holographic plate. The developed holograms are reconstructed using a helium-neon laser and standard pack film. 


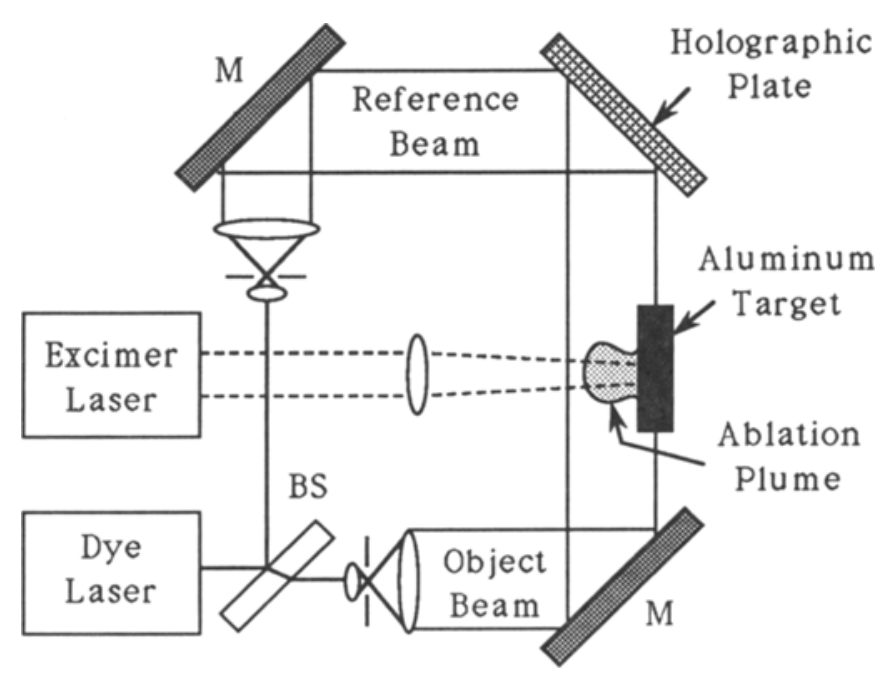

Figure 1. Schematic of experimental configuration

\section{EXPERIMENTAL RESULTS AND INTERPRETATION OF RESONANT INTERFEROGRAMS}

The theory of resonant interferometry was developed by Measures 15 for a Maxwellian velocity distribution of gas atoms, assuming infinitesimal laser linewidth. In Figure 2, the results of Measures theory are given as a response curve of fringes-per-line-density for the ground-state aluminum neutral line at $394.401 \mathrm{~nm}$, assuming zero laser bandwidth. It can be seen that for small wavelength shifts there exists a strong dependence on the temperature of the atoms, but at larger wavelength shifts $(|\Delta \lambda|>0.005$ $\mathrm{nm}$ ) the response curves merge and are insensitive to temperature. For this research, the aluminum neutral plume temperature ${ }^{1,16}$ is assumed to be $0.3 \mathrm{eV}$. It can be shown that the sensitivity of the response curve to laser linewidth is also diminished at larger wavelength shifts. The linewidth of our dye laser without an etalon is $0.2 \mathrm{~cm}^{-1}$. Therefore, we typically operate at larger wavelength shifts $(|\Delta \lambda|>0.006 \mathrm{~nm})$ in order to minimize the effect of laser bandwidth. A theory which includes the effects of laser linewidth is currently being developed. ${ }^{17}$ 


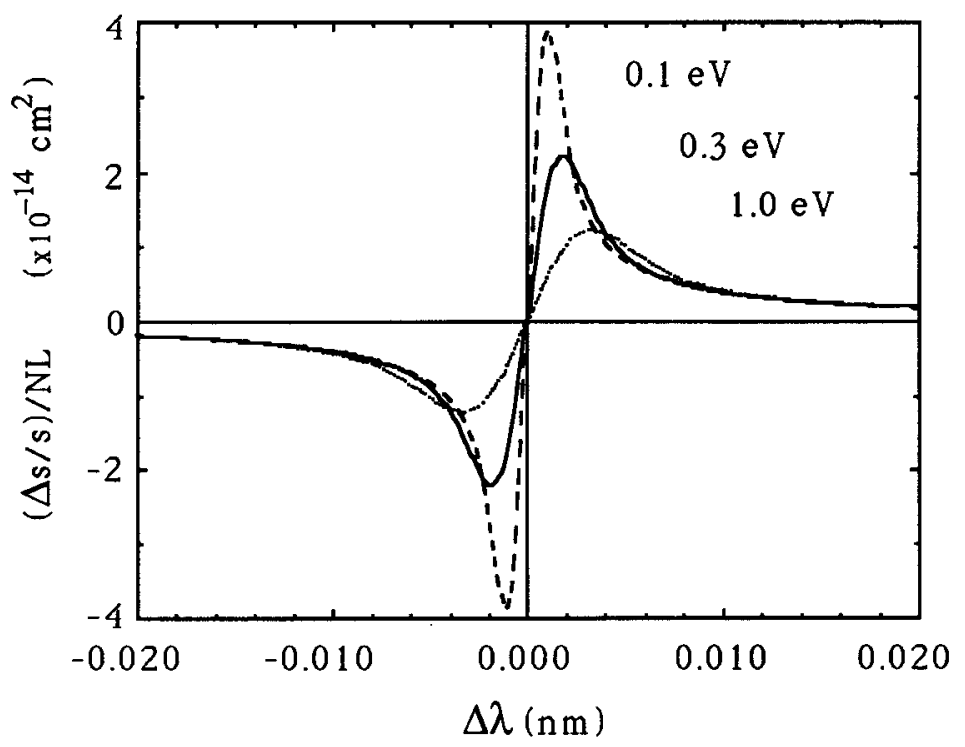

Figure 2. Response curve of fringe shift, $\Delta s / s$, per line density, $N L$, versus dye laser wavelength detuning, $\Delta \lambda$, from $A l$ neutral transition from ground-state at $394.401 \mathrm{~nm}$. From theory of Measures, 15 assuming infinitesimal laser bandwidth.

Figure $3 a$ shows a reconstructed holographic interferogram for laser-ablated aluminum ground-state neutral atoms from an Al metal target at a dye-laser wavelength detuning of $-0.013 \mathrm{~nm}$. Equi-linedensity contours of $\mathrm{Al}$ neutrals are presented in Figure 3b. The peak Al neutral line-density measured in Fig. $3 b$ is about $4.8 \times 10^{14} \mathrm{~cm}^{-2}$. Data for laser ablation in vacuum show rapid Al neutral atom expansion perpendicular to the target, with slower expansion rates parallel to the target. It can be noted that the highest line-densities appear near the target surface, even $0.5 \mu \mathrm{s}$ after the ablation laser pulse has ended.

Laser ablated $\mathrm{Al}$ neutral line-densities from an $\mathrm{Al}_{2} \mathrm{O}_{3}$ target have also been measured by RHI. These results show peak Al neutral line-densities as high as 10 to 20 times larger than the line-densities obtained from Al metal targets. These large ablated Al line-densities from $\mathrm{Al}_{2} \mathrm{O}_{3}$, even at moderate fluences $\left(\sim 4 \mathrm{~J} / \mathrm{cm}^{2}\right)$, could be explained by the research of Dickinson, 18 in which metal atoms are desorbed from defects by intense, ultraviolet laser light.

Resonant holographic interferometry has also been demonstrated in argon gas at 35 Torr, and in a radio-frequency plasma at fill pressures from $30 \mathrm{mTorr}$ to 1 Torr. In the 1 Torr gas, mushroom-cloud-shaped plumes are observed, as seen by others with PLIF. 5 The stem of the mushroom cloud remains attached to the target surface. 

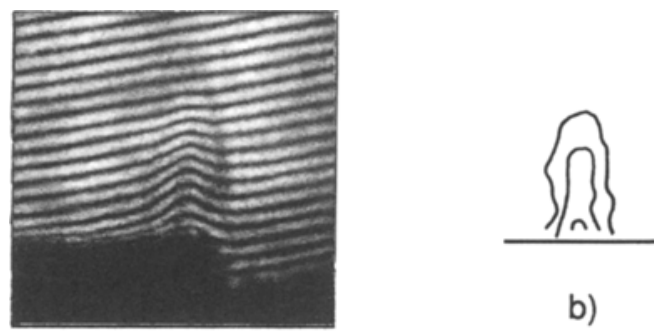

b)

a)

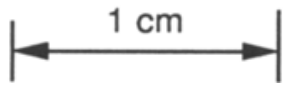

Figure 3. Resonant holographic-interferometry data on laser-ablated Al target in vacuum at fluence of $2.3 \mathrm{~J} / \mathrm{cm}^{2}$, taken at $0.52 \mu \mathrm{s}$ after $\mathrm{KrF}$ laser pulse.

a) Resonant holographic interferogram of ground-state, neutral aluminum, taken at dye-laser wavelength shift of $-0.013 \mathrm{~nm}$.

b) Equi-line-density contours of neutral aluminum obtained from part a. Outer contour represents Al neutral line-density of $1.6 \times 10^{14} \mathrm{~cm}^{-2}$, center contour represents Al neutral line-density of $3.2 \times 10^{14} \mathrm{~cm}^{-2}$, inner contour represents Al neutral line-density of $4.8 \times 10^{14} \mathrm{~cm}^{-2}$.

\section{ACKNOWLEDGEMENTS}

This research is supported by National Science Foundation Grant CTS-9108971. RAL is supported by a DOE MFET Fellowship. JSL is supported by a National Science Foundation Graduate Fellowship.

\section{REFERENCES}

1) R. W. Dreyfus, J. Appl. Phys. $\underline{69} 1722$ (1991)

2) S. C. Langford, J. T. Dickinson, and L. C. Jenson, J. Appl. Phys. $\underline{62}$ 1437 (1987)

3) D. B. Geohegan and D. N. Mashburn, Appl. Phys. Lett. $\underline{55} 2345$ (1989)

4) K. Murakami, in Laser Ablation of Electronic Materials, edited by E. Fogarassy and S. Lazare, Elseviere Science Pub., 1992 
5) A. D. Sappey, T. K. Gamble, and D. K. Zirkle, Appl. Phys. Lett. $\underline{62}$ 564 (1993); also A. D. Sappey and T. K. Gamble, J. Appl. Phys. 725095 (1992)

6) A. Gupta, B. Braren, K. G. Casey, B. W. Hussey, and R. Kelly, Appl. Phys. Lett. 591302 (1991)

7) T. Zyung, H. Kim, J. C. Postwaite, and D. D, Dlott. J. Appl. Phys. 65 4548 (1989)

8) R. M. Gilgenbach and P. L. G. Ventzek, Appl. Phys. Lett. $\underline{58} 1597$ (1991)

9) P. L. G. Ventzek, R. M. Gilgenbach, C. H. Ching, and R. A. Lindley, J. Appl. Phys. 721696 (1992)

10) P. L. G. Ventzek, R. M. Gilgenbach, J. A. Sell, and D. M. Heffelfinger, J. Appl. Phys. 68965 (1990)

11) P. L. G. Ventzek, R. M. Gilgenbach, D. M. Heffelfinger and J. A. Sell, J. Appl. Phys. 70587 (1991)

12) C. L. Enloe, R. M. Gilgenbach, and J. S. Meachum, Rev. Sci. Instrum. 581597 (1987)

13) M. L. Brake, J. Meachum, R. M. Gilgenbach, W. Thornhill, IEEE Trans. Plasma Science, PS-15 73 (1987)

14) R. A. Lindley, R. M. Gilgenbach, and C. H. Ching, Bulletin Am. Phys. Soc. 371453 (1992)

15) R. M. Measures, Appl. Optics, 9737 (1970)

16) D. B. Geohegan, Appl. Phys. Lett. 621463 (1993)

17) R. A. Lindley, Doctoral Dissertation, University of Michigan, Ann Arbor, MI, 1993

18) J. T. Dickinson, S. C. Langford, L. C. Langford, L. C. Jenson, P. A. Eschbach, L. R. Pederson, and D. R. Baer, J. Appl. Phys. $\underline{68} 1831$ (1990) 